\title{
Multimode dynamics in a network with resource mediated coupling
}

\author{
Postnov, D.E.; Sosnovtseva, Olga; Scherbakov, P.; Mosekilde, Erik
}

Published in:

Chaos

Link to article, DOI:

$10.1063 / 1.2805194$

Publication date:

2008

Document Version

Publisher's PDF, also known as Version of record

Link back to DTU Orbit

Citation (APA):

Postnov, D. E., Sosnovtseva, O., Scherbakov, P., \& Mosekilde, E. (2008). Multimode dynamics in a network with resource mediated coupling. Chaos, 18(1), 015114. https://doi.org/10.1063/1.2805194

\section{General rights}

Copyright and moral rights for the publications made accessible in the public portal are retained by the authors and/or other copyright owners and it is a condition of accessing publications that users recognise and abide by the legal requirements associated with these rights.

- Users may download and print one copy of any publication from the public portal for the purpose of private study or research.

- You may not further distribute the material or use it for any profit-making activity or commercial gain

- You may freely distribute the URL identifying the publication in the public portal

If you believe that this document breaches copyright please contact us providing details, and we will remove access to the work immediately and investigate your claim 


\title{
Multimode dynamics in a network with resource mediated coupling
}

\author{
D. E. Postnov \\ Physics Department, Saratov State University, Astrakhanskaya Str. 83, \\ Saratov, 410026, Russia \\ O. V. Sosnovtseva \\ Department of Physics, The Technical University of Denmark, 2800 Kgs. Lyngby, Denmark \\ P. Scherbakov \\ Physics Department, Saratov State University, Astrakhanskaya Str. 83, \\ Saratov, 410026, Russia \\ E. Mosekilde \\ Department of Physics, The Technical University of Denmark, 2800 Kgs. Lyngby, Denmark
}

(Received 7 February 2007; accepted 12 October 2007; published online 27 March 2008)

\begin{abstract}
The purpose of this paper is to study the special forms of multimode dynamics that one can observe in systems with resource-mediated coupling, i.e., systems of self-sustained oscillators in which the coupling takes place via the distribution of primary resources that controls the oscillatory state of the individual unit. With this coupling, a spatially inhomogenous state with mixed high and lowamplitude oscillations in the individual units can arise. To examine generic phenomena associated with this type of interaction we consider a chain of resistively coupled electronic oscillators connected to a common power supply. The two-oscillator system displays antiphase synchronization, and it is interesting to note that two-mode oscillations continue to exist outside of the parameter range in which oscillations occur for the individual unit. At low coupling strengths, the multioscillator system shows high dimensional quasiperiodicity with little tendency for synchronization. At higher coupling strengths, one typically observes spatial clustering involving a few oscillating units. We describe three different scenarios according to which the cluster can slide along the chain as the bias voltage changes. (C) 2008 American Institute of Physics. [DOI: 10.1063/1.2805194]
\end{abstract}

Systems of interacting nonlinear oscillators play an important role in many areas of science and technology. A rat kidney, for instance, contains of the order of $\mathbf{3 0 0 0 0}$ nephrons, each of which tends to display large amplitude oscillations in the regulation of its incoming blood flow. The arteriolar blood pressure controls the oscillatory state of the individual nephron. At the same time, the nephrons interact via the displacement of blood from one nephron to its neighbors produced by this oscillatory regulation. We refer to such structures, in which the oscillatory dynamics of the individual unit can no longer be separated from the coupling among the units, as a system with resource-mediated coupling. To examine some of the generic aspects of this type of system, the paper considers a chain of resistively coupled electronic oscillators sharing a common power supply. For low coupling strengths, the system tends to produce multimode dynamics in the form of high-dimensional quasiperiodicity with little tendency for synchronization. At higher coupling strengths the resource distributed chain displays spatial clustering involving a small number of oscillatory units. In all cases the distribution of individual amplitudes is highly inhomogeneous, showing an interesting example of spatiotemporal mixed-mode oscillations. We study the mechanisms by which this type of cluster slides along the chain as the external bias voltage is changed.

\section{INTRODUCTION}

Initiated by the pioneering work of Winfree, ${ }^{1}$ Kuramoto, ${ }^{2}$ and Kaneko, ${ }^{3}$ the temporal and spatial phenomena that one can observe in systems with large numbers of coupled nonlinear oscillators have attracted an ever growing interest, both from a more mathematical point of view ${ }^{4-6}$ and in view of their relevance to the functioning of various physical, chemical, and biological systems. ${ }^{7-9}$ Traditionally one has considered either lattices of locally coupled oscillators $^{4,10,11}$ with nearest-neighbor interaction or ensembles of globally coupled oscillators ${ }^{12-14}$ with all-to-all interaction. More recently, the concepts of small-world and of scale-free systems have attracted considerable interest. ${ }^{15,16}$

Depending on the boundary conditions, locally coupled systems usually produce standing or traveling waves, including various forms of spatio-temporal chaos. In large ensembles of globally coupled oscillators, the multimode dynamics is often suppressed by the tendency for the oscillators to entrain in states of either global (full) or local (cluster) synchronization. A somewhat related approach, initiated by Rössler, ${ }^{17}$ consists of the systematic development of multimode systems with chaotic dynamics of increasing dimension. This approach may also have interesting applications to a variety of chemical, ecological, and biological systems. ${ }^{18,19}$

The classical synchronization concept ${ }^{20}$ considers the interaction of two or more oscillators, each with their own 
sources of energy, and with the coupling being responsible for the frequency entrainment and mutual amplitude adjustments. For different types of coupling structures (local, global, small-world, etc.), the mathematical description assumes that the nonlinear properties of the individual functional unit (i.e., its natural frequency and/or its resistance to perturbations) are controlled by the unit's own parameters while the interaction is specified through a separate set of parameters characterizing the structure and strengths of the coupling. Hence, one can distinguish the natural modes of the individual oscillators from the properties of the coupling network. Moreover, in most cases the supply of energy to maintain the oscillatory states in the dissipative system is not explicitly represented. To study the effects of synchronization in ensembles of self-sustained units, it is common to use so-called phase oscillators. ${ }^{21}$ Note, however, that this approach cannot be applied in situations where, as in our model, the amplitude variation from unit to unit is large and plays a considerable role in their frequency and phase entrainment.

There are many examples of multimode systems that are composed of a set of individual oscillators connected via a resource distribution network. In such systems, a number of spatially localized oscillatory modes is controlled by the amount of resources (energy) delivered to each individual oscillator and the supply of resources acts as a global control parameter. It is interesting to note that in this case, the initially identical subunits show a tendency for desynchronization, so-called coupling-induced inhomogeneity, combined with a spatial localization of subunits in oscillatory clusters. ${ }^{22}$ The resulting behavior consists of a number of oscillatory modes with different amplitudes and frequencies and can thus be classified as spatiotemporal mixed-mode oscillations. When the control parameters are varied, the spatial location of the oscillatory cluster changes via different forms of mutually related transitions in the individual units.

In the present paper we investigate the above form of multimode dynamics emphasizing an analysis of the underlying nonlinear mechanisms. Having described the individual one-degree-of-freedom oscillator and its electronic implementation, we first investigate how the dynamics of this oscillator is controlled by the power supply. We then consider the main features of the two-mode system to show how competition for energy brings subunits into a state of antiphase synchronization. Finally, we describe the multimode behavior in a large ensemble of oscillators connected via a linear energy distribution network, and explain the underlying nonlinear mechanisms.

\section{INDIVIDUAL OSCILLATOR}

Let us first consider some of the main features of the individual oscillator. As illustrated in Fig. 1(a) this is an electronic resonance circuit with a tunnel diode $D$ in parallel with capacitor. A circuit of this type is commonly used as microwave generator. $L$ and $C$ denote the inductance and capacitance, respectively, and $R_{0}$ is the series resistance. The bias voltage $E_{b}$ plays the role of energy supply for our oscillator system. In a certain range of $E_{b}$, self-sustained oscillations may arise in the system by virtue of the negative dif-
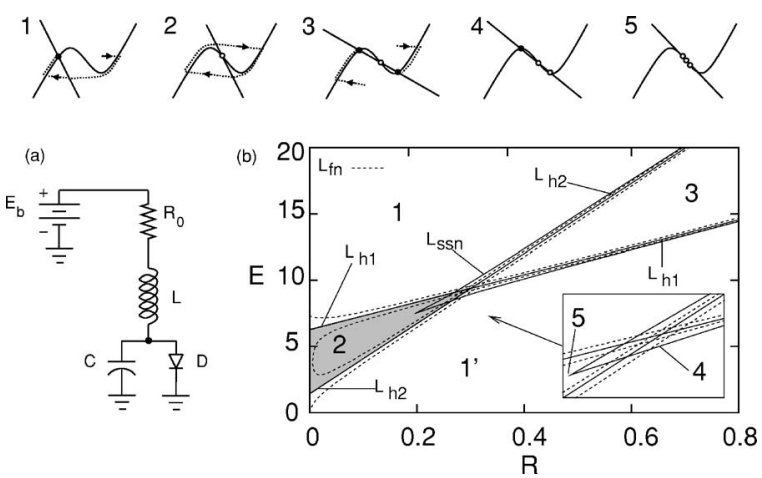

FIG. 1. Electronic oscillator with one degree of freedom. (a) Equivalent circuit diagram. (b) Bifurcation diagram for the model (1) at $\varepsilon=0.1$. Fully drawn lines represent Andronov-Hopf bifurcations, and dotted curves denote transformations of the equilibrium point from node to focus. Insets $\mathbf{1 - 5}$ show the location of the null clines at the points of operation labelled 1-5 in (b), where the filled and open circles denote the stable and unstable equilibrium points, respectively. The gray shaded triangle area corresponds to the region of self-sustained dynamics (the existence of a stable periodic orbit).

ferential resistance associated with the $N$-shaped currentvoltage characteristics of the tunnel diode. The period of these oscillations is determined mainly by the capacitance $C$ and the inductance $L$.

Using Kirchhoff's law for the circuit and introducing the dimensionless time variable $\tau=t / R * C \quad(R *=1 \Omega)$ we can write the governing equations for the resonance circuit in dimensionless form,

$$
\dot{x}=y-f(x), \quad \varepsilon \dot{y}=E-y R-x .
$$

Here, $x$ and $y$ represent, respectively, the voltage across the diode and the current through the inductor. $E$ and $R$ are dimensionless representations of the battery voltage $E_{b}$ and the series resistance $R_{0}$, and $\varepsilon=L / R_{*}^{2} C$ is a scaling factor that separates the fast and slow dynamics of the oscillator. In the following, $\varepsilon=0.05$. The current-voltage characteristics of the tunnel diode $f(x)$ is assumed to be of cubic shape with a nonlinearity of the form,

$$
f(x)=20 x-5 x^{2}+x^{3} / 3 .
$$

With these equations, our 2D oscillator is similar to the FitzHugh-Nagumo model, often used as a simple example of a nerve model. ${ }^{23}$ However, the choice of control parameters is different. Figure 1(b) shows the regions of distinct operating conditions with the insets illustrating the corresponding locations of the null clines for the system. Note that the $x$ null cline coincides with $f(x)$ while the $y$ null cline, defined by $y=(E-x) / R$ and sometimes called the load line, is a linear function with slope $1 / R$.

Let us first consider the behavior for small values of $R$. In this regime, intersection of the null clines may occur outside of the interval with negative slope of $f(x)$ (inset 1), i.e., both for small and for large values of $E$ there is a single, stable equilibrium point, or the intersection may take place in the region of negative slope for the $x$ null cline (inset 2).

For sufficiently small values of $\varepsilon$, intersection of the null clines at the local maximum or minimum of $f(x)$ determines the points of Andronov-Hopf bifurcation. Such bifurcations occur at the lines labelled $L_{h 1}$ and $L_{h 2}$. Approaching these 


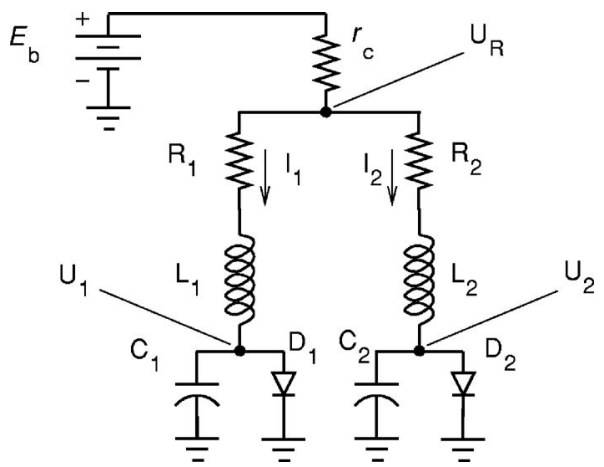

FIG. 2. Circuit diagram for two oscillators coupled via a shared power supply (battery). The coupling strength is controlled by the resistor $r_{c}$. The interesting feature of this diagram is that the coupling takes place via the network that controls the dynamic state of the individual oscillator. In our dimensionless equations, $r_{c}$ and $U_{R}$ are represented by $r$ and $U$, respectively.

lines from both sides, the equilibrium point transforms from a node to a focus at the dashed lines $L_{f n}$. Within the gray area labeled 2 there is a single unstable equilibrium point at the segment of $f(x)$ with negative slope. This is the region of self-sustained oscillations, and the corresponding limit cycle trajectory is schematically depicted in the inset $\mathbf{2}$.

At large values of $R$, when the slope of the $y$ null cline is small, there are three points of intersection with the cubic $x$ null cline (the area and inset labelled 3 ). This implies the presence of two stable and one unstable equilibrium points. Hence, the system is bistable and does not show selfsustained dynamics. When one approaches this area from small or large $E$ values, two consecutive events take place. The first is the appearance of a pair of unstable points at the line of saddle-node bifurcation $L_{s s n}$. One of these points is a saddle while the other is an unstable node. The second event is that the unstable node becomes a focus on the dashed line, undergoes an inverse Andronov-Hopf bifurcation, and then becomes a stable node at the second dashed line.

At intermediate values $0.2<R<0.4$ the two null clines have approximately equal slope in the vicinity of their intersection and the area between the bifurcation lines shrinks. The enlargement shown in Fig. 1(b) reveals the details and illustrates the saddle-saddle-node bifurcation curve $\mathbf{4}$ as well as codimension-two bifurcation $\mathbf{5}$ when (i) the $y$ null cline crosses through the center of the cubic $x$ null cline and (ii) the slopes of both null clines at this point are equal.

To summarize, the main dynamical regimes of the individual oscillator (1) are the excitable regime $\mathbf{1}$ (and $\mathbf{1}^{\prime}$ which is symmetric to $\mathbf{1}$ with respect to the center of the cubic function), the region of self-sustained dynamics $\mathbf{2}$, and the bistable regime 3 . Note that the region of self-sustained oscillatory behavior existing at low values of $R$ is limited. For a given value of $R$, oscillations do not arise neither at too high nor at too low values of the supplied energy (as represented by the battery voltage $E$ ).

\section{DYNAMICAL PATTERNS IN THE TWO-MODE SYSTEM}

Let us examine the dynamics of two coupled units. Figure 2 shows the circuit diagram for two oscillators that are connected to the battery via the common resistor $r_{c}$. The corresponding model equations can be easily obtained by copying Eq. (1) for the second system (denoting the two oscillators with subscripts 1 and 2) and accounting for the voltage drop across the resistor $r_{c}$,

$$
\begin{aligned}
& \dot{x}_{1,2}=y_{1,2}-f\left(x_{1,2}\right), \\
& \varepsilon_{1,2} \dot{y}_{1,2}=\left\{E-r\left(y_{1}+y_{2}\right)\right\}-R_{1,2} y_{1,2}-x_{1,2} .
\end{aligned}
$$

An overview of the dynamical regimes for two identical diode oscillators on the $(r, E)$ parameter plane is presented in Fig. 3(a). Here, the vertical direction represents variation of the power supply while the horizontal direction corresponds to changes of the coupling strength. Typical behaviors are depicted in the diagram insets in the form of sketches of $\left(x_{1}, x_{2}\right)$ phase projections. The main feature of the observed behaviors is an antiphase locking pattern illustrated by a backwards sloping figure-8. In several regions, this antiphase regime coexists with one or two stable equilibrium points. The appearance of antiphase oscillations can be explained in terms of the structure of the equations: One can rearrange Eqs. (3) by grouping terms with $y_{1}$ or $y_{2}$. In this way, $\left(R_{1,2}\right.$ $+r$ ) becomes equivalent to the resistance of the individual circuit in Fig. 1, and the coupling term becomes $-r y_{2,1}$. Thus, increasing $y_{1}$ causes a drop of $y_{2}$ and vice versa. From a
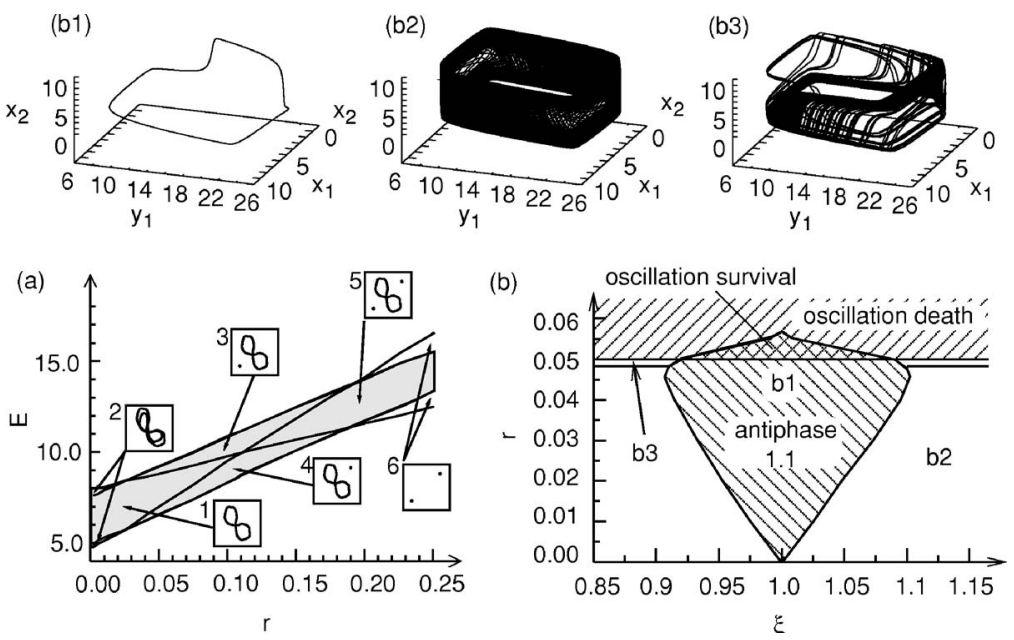

FIG. 3. Overview of dynamical regimes of two coupled oscillators. (a) Sketch of the bifurcation diagram on the $(r, E)$ parameter plane for identical oscillators. (b) Diagram on the $(\xi, r)$ parameter plane with the frequency mismatch $\xi=\epsilon_{1} / \epsilon_{2}$. Insets b1-b3 show 3D phase projections for the regions labelled b1-b3 in (b). 
more generic point of view, we consider a system with competitive coupling. This means that increasing the current to one oscillator reduces the current to the other and that each of the interacting units tries to prevent the other unit from following its motion. In biological systems, examples of this type of coupling may be found, for instance, for competing bacterial populations ${ }^{9}$ or for tubular pressure oscillations of paired nephrons. $^{24}$

Note that, according to Eq. (3), increasing $r$ acts for each unit in the same way as increasing $R$ in Eq. (1). Hence, the bifurcation diagram contains two crossing lines of Andronov-Hopf bifurcation similar to those in Fig. 1(b). However, the area of self-sustained dynamics (indicated in gray) is no longer bounded by these lines. Qualitatively this can be explained as follows. When an individual unit is in an oscillatory state, the current through the inductor changes its direction twice per period. When two circuits are coupled as shown in Fig. 2, current will be displaced from one circuit to the other and, in spite of the competitive character of the coupling, the interacting units thus help one another to maintain the oscillations. This is possible only for antiphase locking, where the currents in the coupled units have opposite phase.

The dynamical pattern described above is preserved in some range of the mismatch parameter. Figure 3(b) illustrates synchronous and nonsynchronous regimes on the parameter plane of the frequency mismatch $\xi=\varepsilon_{2} / \varepsilon_{1}$ and the coupling parameter $r$. The Arnol'd tongue for antiphase locking (regime b1) emanates from the point $\xi=1.0, r=0$. Increasing $r$ strengthens the coupling and, thus, the synchronization region becomes wider. Above $r \approx 0.05$, the asynchronous oscillators do not demonstrate self-sustained dynamics. Inside the synchronization region, however, selfsustained dynamics is maintained for larger values of $r$. Note that before the asynchronous oscillations $\mathbf{b} 2$ die, they pass through a chaotic regime labeled as $\mathbf{b 3}$. This occurs when the units force each other close to the Andronov-Hopf bifurcation where the divergence of the phase flow is high. ${ }^{25,26}$ This phenomenon is related to the existence of an area of canard explosion. $^{27,28}$

Let us summarize our findings concerning the behavior of the two-oscillator system: (i) sharing the common power supply via a resistor, identical coupled circuits tend to attain antiphase synchronization; (ii) the antiphase locking pattern may maintain self-sustained dynamics even outside of the corresponding parameter range for the individual system; and (iii) this effect is preserved in the presence of a mismatch of reasonable size between the coupled units.

\section{MULTIMODE NETWORK}

To build a multimode system of functional units (1) we connect the oscillators as shown in Fig. 4. We have previously introduced such a coupling structure as a simple implementation of a system with resource mediated coupling. ${ }^{22}$ Capacitors $C_{z}$ are introduced to account for possible accumulation of energy by an individual oscillator while the resistors $r_{c}$ are responsible for the finite replenishment rate and for transmission losses. Values of $R_{j}, L_{j}, C_{j}$ are assumed to be the same for all units. Now the $j$ th individual oscillator is fed

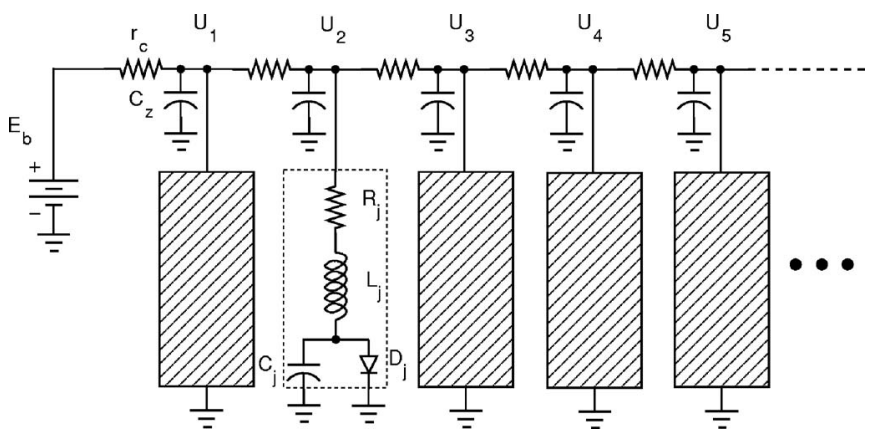

FIG. 4. Circuit diagram for an array of oscillators with the geometry of a linear power distribution chain.

from the corresponding branching point with voltage $U_{j}$. To account for this coupling structure in a dimensionless model we introduce the new variable $z_{j}$ defined for each branching point and representing the corresponding voltage $U_{j}$. The modified equations for each oscillator then reads

$$
\begin{aligned}
& \dot{\gamma z_{j}}=\left(z_{j-1}-z_{j}\right) / r+\left(z_{j+1}-z_{j}\right) / r-y_{j}, \quad j=1, \ldots, N, \\
& \dot{x}_{j}=y_{j}-f\left(x_{j}\right), \quad \varepsilon \dot{y}_{j}=z_{j}-y_{j} R-x_{j},
\end{aligned}
$$

where $\gamma=C_{z} / C_{j} . j$ represents the number of the oscillator, and $N$ is the total number of units. $z_{0}$ corresponds to the battery voltage $E$. The free end of the chain is modeled by the boundary condition $z_{N+1}=z_{N}$. We assume that $C_{z} \ll C_{j}$ so that $\gamma \ll 1$ and $z_{j}$ quickly follows variations of $x_{j}$ and $y_{j}$.

Organized in the chain (4), the units become globally coupled via variations of the $z_{j}$ variables. There is a gradual decrease of the mean value of $z_{j}$ along the chain because of the voltage drop across each coupling resistor $r$. Note that the current along the chain splits into two currents at each unit. Thus the current decreases along the chain, and the drop of $z_{j}$ from unit to unit becomes smaller and smaller. In our original model of a chain of microbiological population pools, this gradual decrease of the "energy" available to the individual oscillator could be compensated by additional supplies of substrate to the various pools. In this way the oscillations could be maintained all the way along the chain. A similar supplementary feed structure appears less natural for a chain of electronic oscillators.

In the phase space of the whole system, the variation of the mean value of $z_{j}$ affects the stability of the global equilibrium state that can be defined from

$$
\dot{x}_{j}=0, \quad \dot{y}_{j}=0, \quad \dot{z}_{j}=0, \quad j=1, \ldots, N .
$$

We have previously shown ${ }^{22}$ that, under variation of $E$, a sequence of Andronov-Hopf bifurcations occurs in the phase subspaces of each array unit. For a given value of $E$ there is some number of oscillators (generally less than $N$ ) with unstable equilibrium points and, thus, show self-sustained dynamics. This leads to the formation of spatially localized multimode behavior, referred as an oscillatory cluster, that can slide along the array with variations of $E$.

Below we consider an array of ten units and focus on (i) the frequency patterns associated with multimode oscillatory 

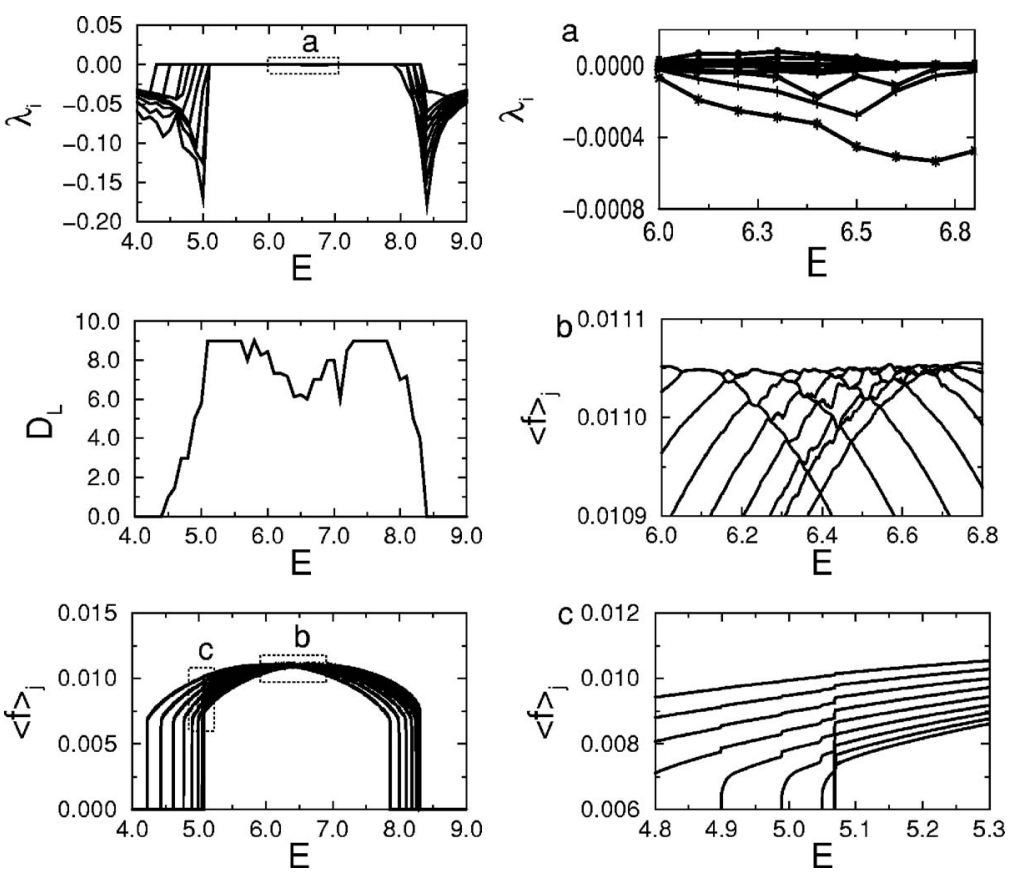

FIG. 5. Formation of multifrequency regimes in a power sharing array of ten oscillators. Left panels show plots of Lyapunov exponents (top), the corresponding changes in Lyapunov dimension $D_{L}$ (middle), and plots of frequencies for the various oscillators (bottom). Right panels represent enlargements of the areas labeled $a, b$, and $c$. Calculations were performed for $\varepsilon=0.1, r$ $=0.05, \gamma=0.001$, and $R=0.001$. clusters and (ii) the transition scenarios that can be observed when a cluster changes its position along the chain.

\section{A. Weak coupling}

The choice of $r=0.05, \varepsilon=0.1, \gamma=0.001$, and $R=0.01$ provides a weak interaction between the units of the array. The multimode oscillatory patterns that arise in the chain will be characterized by the ten largest Lyapunov exponents $\lambda_{i}$, the corresponding Lyapunov dimension $D_{L}$, and the individual frequencies $\langle f\rangle_{j}$, calculated from mean return time to Poincaré sections defined by the condition $x_{j}=5.0$ in Fig. 5 .

Note that the Lyapunov exponents $\lambda_{i}$ have the subscript $i=1, \ldots, 3 N$. Formally it is not possible for a system with global coupling to establish a direct relation between $\lambda_{i}$ and specific oscillator units. However, it is possible to associate each zero-valued Lyapunov exponent with one of the individual oscillators. In this way, we discuss the plots for the $N$ largest Lyapunov exponents $\lambda_{i}$ and for the $N$ frequencies $\langle f\rangle_{j}$ together.

As the figure shows, increasing $E$ from 4.3 to 5.2 is associated with the Lyapunov exponents one by one approaching zero from below (top left panel in Fig. 5). The Lyapunov dimension rises up to $D_{L}=9.0$. The corresponding frequencies appear and rise gradually without visible locking regions (bottom left panel). This indicates the formation of multimode behavior represented in phase space by a highdimensional torus with a number of independent frequencies equal to the number of vanishing Lyapunov exponents. A detailed inspection of the figures for $E \in[6.0 ; 6.8]$ shows the presence of small regions of partial resonances and corresponding weakly negative Lyapunov exponents (insets a and b). The individual frequencies are close enough to one another that the interaction via the power distribution gives rise to locking patterns.

Within the range of $E$ shown in inset c we cannot distinguish any partial resonances. If one assumes the presence of higher-order resonances such as $p\left\langle f_{j}\right\rangle=q\left(\left\langle f_{j+k}\right\rangle, j+k<N\right)$, where $p$ and $q$ are integers, then the higher frequency should rise faster than the other. This appears not to be the case in Fig. 5. We can conclude that for certain sets of parameters the system under consideration shows oscillations with up to nine independent frequencies, or the equivalent of a ninedimensional torus. In the inset $\mathbf{c}$, sharp steps are clearly distinguished in the frequency curves. We leave the explanation of this behavior till the last section of the paper.

\section{B. Strong coupling}

With increasing $r$ the voltage drop between the branching points increases, and only a few oscillators show selfsustained dynamics. At $r=0.274$ the oscillatory cluster shrinks to only two units, and the values of $E$ must be considerably larger to observe different cluster locations along the chain.

Figure 6 shows the location of the cluster (middle panel), the frequencies of the oscillating units (top panel) and the values of the largest Lyapunov exponents (bottom panel). For most values of $E$ the oscillatory cluster contains two units. At $E=10.10$, self-sustained dynamics are observed in units 1 and 2 . The heavy black circles in the middle panel indicate the particular parameter values used for the phase projections in the respective insets. With increasing $E$, sequential changes of cluster location to positions 2-3, 3-4, 4-5-6, and 7-8-9-10 are observed. In the following we consider typical regimes in the center range of cluster stability (with respect to values of $E$ ) together with different scenarios observed for the shift in cluster position.

\section{Intracluster synchronization}

Figure 6 clearly demonstrates the region of frequency locking for each cluster location. This region may also be diagnosed by the characteristic gap in the plot for Lyapunov exponents. Examples of phase projections are shown in insets $\mathbf{b}$ and $\mathbf{c}$. 


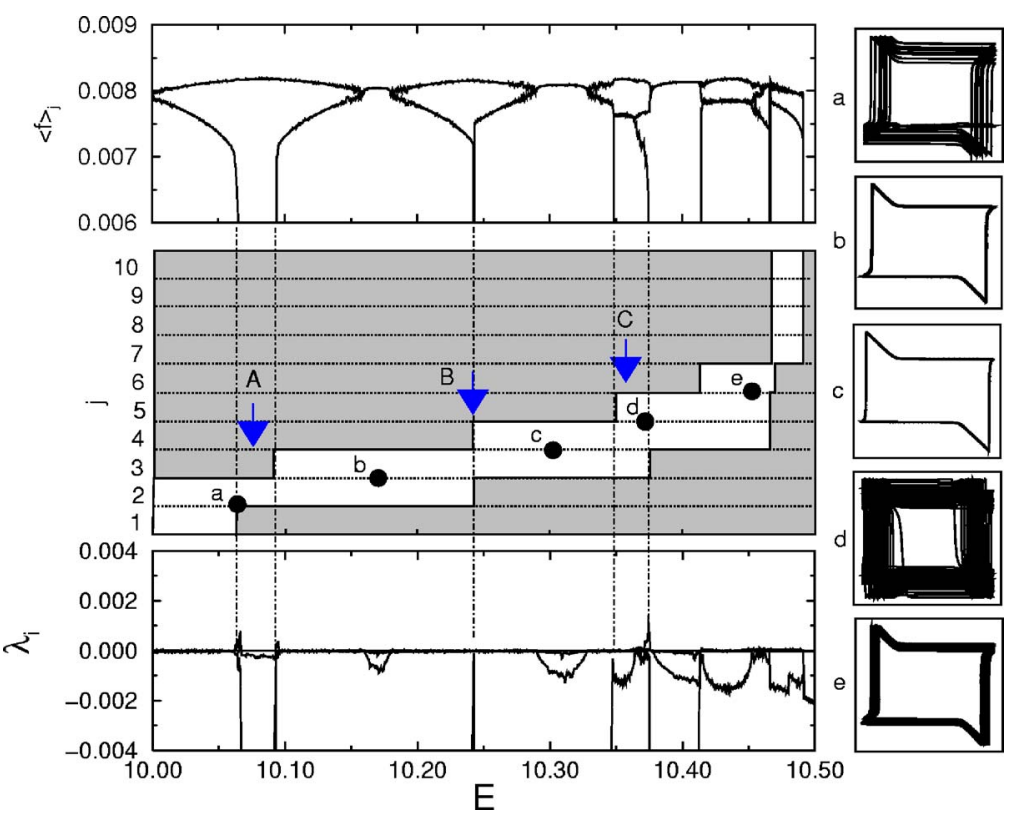

FIG. 6. (Color online) Variations of the oscillatory regimes with the bias voltage $E$ at strong coupling $r$ $=0.274$. The middle panel (in gray) shows the location of a two-mode oscillatory cluster. The top and bottom panels show the corresponding changes in frequencies and Lyapunov exponents, respectively. The insets (a) (e) show the phase projection on the $\left(x_{j}, x_{j+1}\right)$ plane, $(j, j+1)$ is defined by the location of the corresponding filled circle in the central panel. Other parameters are fixed at $\varepsilon=0.1, \gamma=0.001$, and $R=0.001$.
Figure 7 shows enlargements of the dependences of the two largest Lyapunov exponents $\lambda_{j}$ and of the phase difference $\Delta \phi$ on $E$. As one can see, the value of $\Delta \phi$ varies approximately from $\pi / 2$ to $3 \pi / 2$. This means that the observed synchronous regime is closer to antiphase than to in-phase synchronization. A pure antiphase locking pattern with $\Delta \phi$ $=\pi$ is observed for $E \approx 10.314$. Note that this value of $E$ does not correspond precisely to the maximal stability of the synchronous regime (which occurs at the minimal value of the negative Lyapunov exponent or at $E \approx 10.311$ ). This observation can be explained by the results described in Sec. III. Namely, when the cluster consists of only two self-sustained units, the remaining oscillators, that are in the damped state, can be considered as passive elements and, thus, replaced by equivalent resistors. Hence, the behavior of the ten-unit array in this regime is similar to the behavior of two coupled

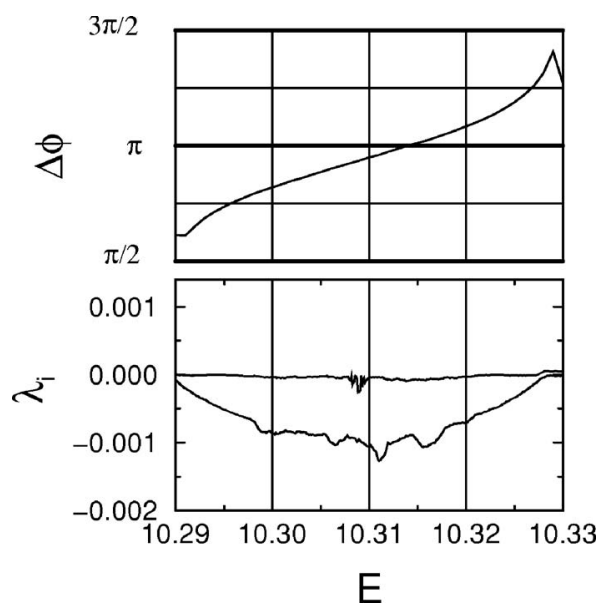

FIG. 7. The top plot shows the phase difference $\Delta \phi$ vs the bias voltage $E$ across a tongue with antiphase synchronization. The bottom panel represents the corresponding variations of the two largest Lyapunov exponents. oscillators in the antiphase locking regime. However, such an equivalent system is generally not as symmetric as the system considered in Sec. III. This explains the observed shift in the location of purely antiphase regime with respect to the local minimum of the Lyapunov exponents.

\section{Change of cluster location}

We have observed three different mechanisms by which the synchronized cluster can shift its position along the chain.

Scenario A: In the range of $E \in[10.05 ; 10.10]$ the oscillatory cluster changes its location from units $1-2$ to units $2-3$ (Fig. 6). This occurs in two steps: (i) unit 1 ceases to oscillate at $E=10.0658$ and (ii) unit 3 starts to oscillate at $E$ $=10.0929$. In the interval between the two values of $E$ the cluster consists of only a single oscillator. Figure 8 (left panel) shows the detailed plot of the largest Lyapunov exponents. The dashed circles focus on the most interesting aspect of the considered transition. Namely, both extinction of oscillations in the first unit, and the onset of oscillations in the third unit are accompanied by bursts of the Lyapunov exponents to positive values, indicating a narrow chaotic regime. Inspection of phase projections helps to reveal the responsible mechanism. There are no particularly interesting features of the phase projection on the $\left(x_{2}, y_{2}\right)$ plane. However, the phase plot on the $\left(x_{1}, y_{1}\right)$ plane shows chaotic behavior [Fig. 8 (right panel)]. An enlargement of the area close to the equilibrium state reveals the characteristic bundle of trajectories. Thus, the periodic forcing by the second unit drives the first unit across the Andronov-Hopf bifurcation point. As already mentioned, our model (1) is similar to the wellknown FitzHugh-Nagumo model that demonstrates the specific feature known as a canard explosion near the supercritical Andronov-Hopf bifurcation. ${ }^{27,28}$ This is related to a local but strong divergence of the trajectories. Note that to observe the canard explosion the time separation parameter $\varepsilon$ should be small. When forced by external regular or noisy signals, a 

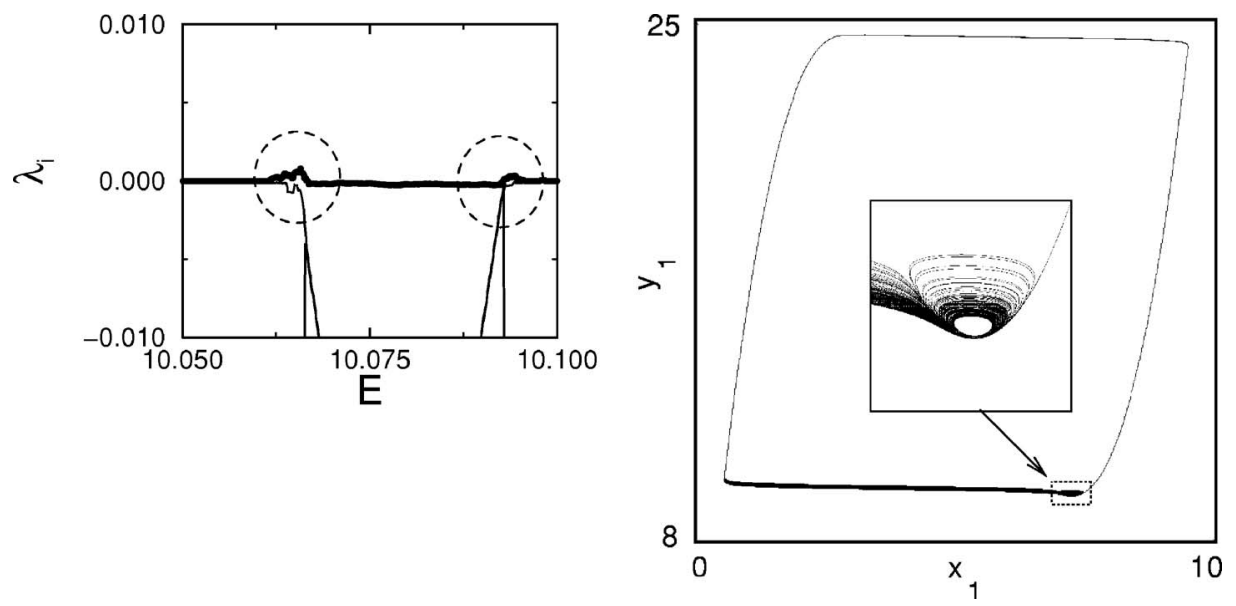

FIG. 8. Enlargement of part of the bottom panel in Fig. 6 around the region labeled $A$ (left panel), phase projection on the $\left(x_{1}, y_{1}\right)$ plane, and its enlargement (right panel). system in the canard region is known to produce chaos. $^{25,26,29}$

Hence, the scenario A for changes of cluster location includes two regions of chaotic behavior related to the extinction and to the onset of oscillations in edge units.

Scenario B: The above discussion suggests that the transition of any unit of the array through an Andronov-Hopf bifurcation should be accompanied by the appearance of a chaotic regime. However, this is not the case for the transition B (Fig. 6). The plot for the largest Lyapunov exponents [Fig. 9 (left panel)] shows that the second Lyapunov exponent reaches zero at the single point $E=10.2423$. At this point the oscillatory cluster abruptly changes its location. Note that the shift of cluster position involves two different events. First, the extinction of oscillations in the "tail" unit of the cluster. Second, the onset of oscillations in the "head" unit. When we observe both events at the same value of parameter $E$ the regime should be structurally unstable. The diagram of dynamic regimes in Fig. 9 (right panel) clarifies the situation. From the diagram one can see the disposition of regions with different cluster locations. It is clearly seen that the observed "jump" of the cluster according to the scenario $\mathbf{B}$ is structurally stable but associated with a regime of bistability where the different cluster locations at units 2-3 and 3-4 coexist. Similar but more narrow areas of bistability can be found at the top and bottom of the diagram. They are associated with transitions through cluster narrowing and widening, respectively.

The key question is why such behavior patterns occur while the individual unit does not show any bistability? As shown in Sec. II, both the onset and the extinction of oscillations in the model (1) are associated with a soft (or supercritical) Andronov-Hopf bifurcation. To explain this we build the bifurcation diagram on the $(E, y)$ parameter plane and plot the mean value of $y$ averaged over the period of oscillations. Note that in terms of the original electronic circuit (Fig. 1) the $y$ variable describes the current through the inductor $L$. As one can see, the onset of oscillations [Fig. $10(a)]$ causes a sharp drop in the mean consumed current $\langle y\rangle$. In turn, this drop in current consumption increases the voltage $U_{j}$ in all branching points (Fig. 4). Thus, if, for example, the third unit enters the self-sustained regime, then the voltage increases on all units, including the third unit itself. In this way, its operating point is shifted as if $E$ was increased. This causes the abrupt "jumps" to the regime of full-range oscillations, skipping the chaotic regime discussed for the scenario A. In Fig. 10(b) the described shift of the operating point is schematically depicted with points labeled as 1 $\rightarrow 1$ '. For the "tail" unit of the cluster, where the oscillations die out, $\langle y\rangle$ changes in a similar way: the transition causes a drop of the mean consumed current that in turn "pushes" the unit out of self-sustained regime. This transition is denoted $2 \rightarrow 2$ ' in Fig. 10(b). The described mechanism also explains the sharp steps in frequency curves observed in Fig. 5(c).

Scenario C: The first two mechanisms for change of cluster location can be realized in combination. Figure 11 represents the plot for the largest Lyapunov exponents in the range of bias voltage $E$ corresponding to the transition $\mathbf{C}$ in Fig. 6. One can see that the fourth unit enters the self-
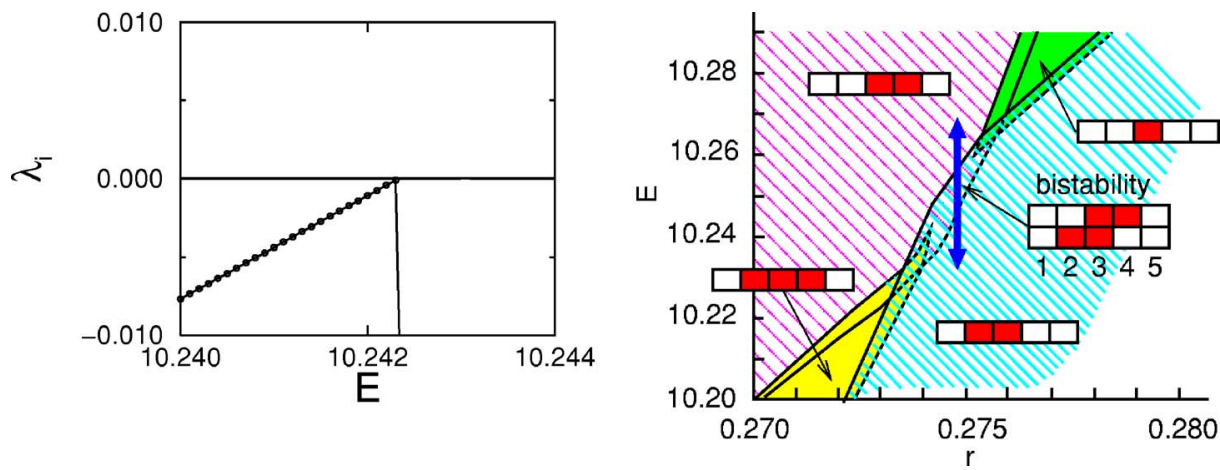

FIG. 9. (Color online) Abrupt change of cluster location (transition $B$ in Fig. 6). Plot for the Lyapunov exponents $\lambda_{1,2}$ shows the single point where $\lambda_{1}$ $=\lambda_{2}=0$ (left panel). The diagram of regimes on the $(r, E)$ plane shows the stability of different regimes (right panel). The rectangle insets in the diagram schematically show the first five units where the self-sustained regime is indicated by the gray shading. 

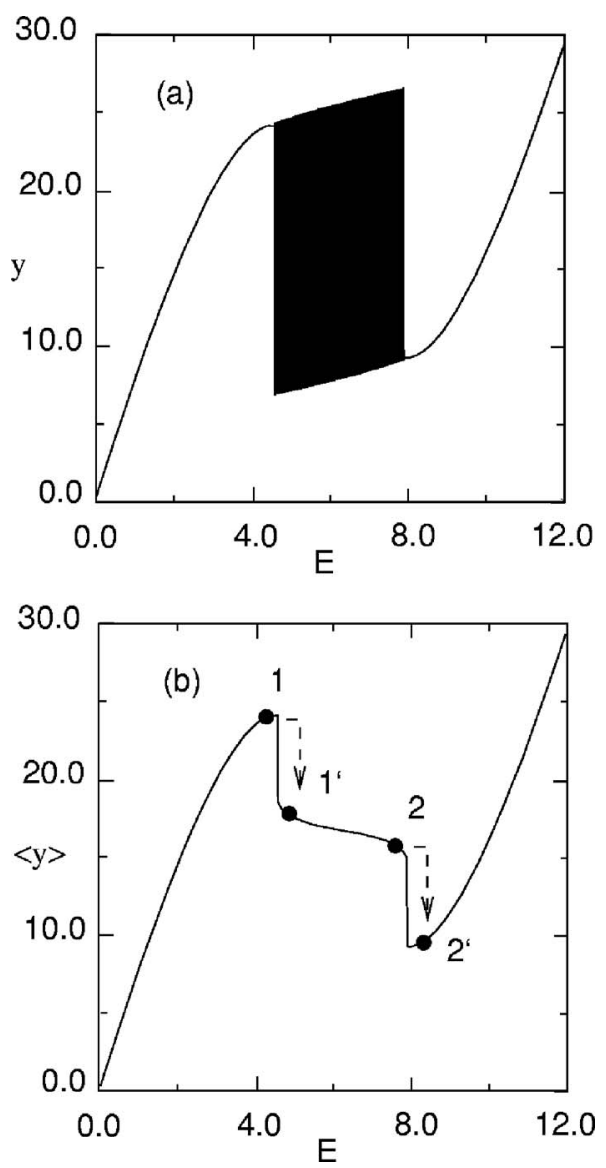

FIG. 10. The features of the individual model (1) that reflects the current consumption by the electronic circuit in Fig. 1. (a) The bifurcation diagram shows the location of the equilibrium point and the range of self-sustained oscillations. (b) Corresponding variation of the mean value of the current consumption $\langle y\rangle$ averaged over the period.

sustained regime according to scenario $B$ at $E=10.3471$. At larger $E \in[10.3699 ; 10.3748]$ a chaotic regime appears that is associated with extinction of the self-sustained regime in the third unit.

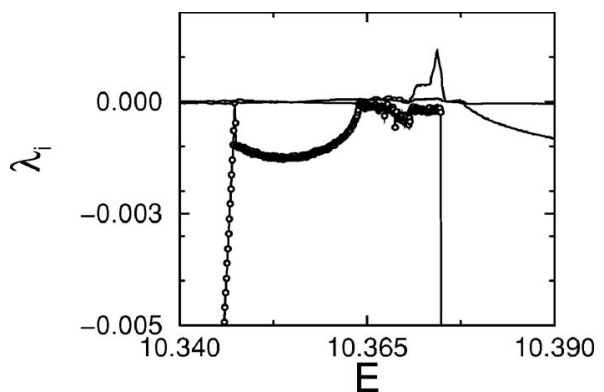

FIG. 11. Plot of the largest Lyapunov exponents for the scenario $C$ in Fig. 6. Here the head unit in the cluster enters the regime of self-sustained oscillations in accordance with scenario B (Fig. 9) while extinction of selfsustained oscillations for the tail unit proceeds along scenario $\mathbf{A}$.

\section{v. CONCLUSIONS}

Considering an array of resistively coupled electronic oscillators we examined a particular form of multimode dynamics, referred to as spatial oscillator clustering. This type of multimode dynamics can be observed in systems in which the energy supply (or resource allocation) that controls the dynamic state of the individual oscillator also provides the mechanism of coupling among the oscillators. The result is a spatially inhomogeneous state of self-sustained oscillations involving a variable number of modes and shifting along the system in dependence of the total supply of resources. We analyzed the frequency pattern within such a cluster and outlined three possible scenarios of cluster displacement, i.e., of spatial reorganization of the multimode behavior. Our main findings can be summarized as follows:

- Sharing a common power supply via some resistor, two identical coupled oscillator circuits tend to lock in an antiphase mode that supports the self-sustained dynamics in the whole system even outside the parameter range of oscillations for the individual system.

- A linear array of electronic oscillators shows spatially localized multimode dynamics in the form of an oscillatory cluster. The voltage drop from one branching point to another is responsible for the behavioral inhomogeneity of the originally identical units and, hence, underlies the formation of the oscillatory cluster.

- For weak coupling (small voltage drop from one branching point to the next) quasiperiodic behavior with a variable number of independent frequencies (each representing one oscillatory mode) is found to be the characteristic operating regime. The numerical simulations show an interval of the control parameters where an up to nine-mode quasiperiodic regime is observed without pronounced tendency for mode locking or torus destruction.

- At stronger coupling (strong voltage drop from one branching point to the next) the cluster shrinks to a few units (two- or three-mode operating regimes) and shows several different scenarios for the cluster displacement under variation of the power supply. These scenarios include chaos development in the edge elements of the cluster as well as coexistence of different spatial patterns (different cluster locations). We explain the formation of bistability in terms of coupling-induced positive feedback and the nonlinear nature of the mean current consumption by the individual oscillatory unit.

\section{ACKNOWLEDGMENTS}

This work was supported by RFBR Grant No. 04-0216769 and by the European Union through the Network of Excellence BioSim (Contract No. LSHB-CT-2004-005137).

\footnotetext{
${ }^{1}$ A. T. Winfree, "Biological rhythms and the behavior of populations of coupled oscillators," J. Theor. Biol. 16, 15 (1967).

${ }^{2}$ Y. Kuramoto, Chemical Oscillations, Waves and Turbulence (Springer, Berlin, 1984).
} 
${ }^{3}$ K. Kaneko, "Spatial period-doubling in open flow," Phys. Lett. 111, 321 (1985).

${ }^{4}$ S. P. Kuznetsov, in Theory and Applications of Coupled Map Lattices, edited by K. Kaneko (Wiley, New York, 1993).

${ }^{5}$ O. A. Druzhinin and A. S. Mikhailov, "Regular and chaotic spatial patterns in a coupled map lattice," Phys. Lett. A 148, 429 (1990).

${ }^{6}$ V. S. Afraimovich and V. S. Nekorkin, "Chaos of traveling waves in a discrete chain of diffusively coupled maps," Int. J. Bifurcation Chaos Appl. Sci. Eng. 4, 631 (1994).

${ }^{7}$ P. Hadley, M. R. Beasley, and K. Wiesenfeld, "Phase locking of Josephson junction series arrays," Phys. Rev. B 38, 8712 (1988).

${ }^{8}$ H. D. I. Abarbanel, A. Selverston, R. Huert, M. V. Bazhenov, M. M. Sushchik, L. L. Rubchinskii, and M. I. Rabinovich, "Synchronization in neuron ensembles," Usp. Fiz. Nauk 166, 363 (1990) [Sov. Phys. Usp. 39, 337 (1990)].

${ }^{9}$ E. Mosekilde, Topics in Nonlinear Dynamics: Applications to Physics, Biology and Economic Systems (World Scientific, Singapore, 1996).

${ }^{10}$ V. N. Belykh and E. Mosekilde, "One-dimensional map lattices: Synchronization, bifurcations, and chaotic structures," Phys. Rev. E 54, 3196 (1996).

${ }^{11}$ G. V. Osipov, A. S. Pikovsky, M. G. Rosenblum, and J. Kurths, "Phase synchronization effects in a lattice of nonidentical Rössler oscillators," Phys. Rev. E 55, 2353 (1997).

${ }^{12}$ K. Kaneko, "Globally coupled circle maps," Physica D 54, 5 (1991).

${ }^{13}$ O. Popovych, Yu. Maistrenko, and E. Mosekilde, "Loss of coherence in a system of globally coupled maps," Phys. Rev. E 64, 026205 (2001).

${ }^{14}$ S. De Monte, F. D'Ovidio, and E. Mosekilde, "Coherent regimes of globally coupled dynamical systems," Phys. Rev. Lett. 90, 054103 (2003).

${ }^{15}$ S. H. Strogatz, "Exploring complex networks," Nature (London) 410, 268 (2001).

${ }^{16}$ V. Latora and M. Marchiori, "Efficient behavior of small-world networks," Phys. Rev. Lett. 87, 198701 (2001).
${ }^{17}$ O. E. Rössler, "The chaotic hierarchy," Z. Naturforsch. A 38A, 788 (1983)

${ }^{18}$ G. Baier, J. S. Thomsen, and E. Mosekilde, "Chaotic hierarchy in a model of competing populations," J. Theor. Biol. 165, 593 (1993).

${ }^{19}$ D. E. Postnov, A. G. Balanov, O. V. Sosnovtseva, and E. Mosekilde, "Chaotic hierarchy in high dimensions," Int. J. Mod. Phys. A 14, 2511 (2000).

${ }^{20}$ A. Pikovsky, M. Rosenblum, and J. Kurths, Synchronization: A Universal Concept in Nonlinear Sciences (Cambridge University Press, Cambridge, 2001).

${ }^{21}$ G. V. Osipov and J. Kurths, "Regular and chaotic phase synchronization of coupled circle maps," Phys. Rev. E 65, 016216 (2002).

${ }^{22}$ D. E. Postnov, O. V. Sosnovtseva, and E. Mosekilde, "Oscillator clustering in a resource distribution chain," Chaos 15, 013704 (2005).

${ }^{23}$ R. A. FitzHugh, "Impulses and physiological states in theoretical models of nerve membrane," Biophys. J. 1, 445 (1961).

${ }^{24}$ D. E. Postnov, O. V. Sosnovtseva, E. Mosekilde, and N.-H. HolsteinRathlou, "Cooperative phase dynamics in coupled nephrons," Int. J. Mod. Phys. B 15, 3079 (2001).

${ }^{25} \mathrm{M}$. Sekikawa, N. Inaba, and T. Tsubouchi, "Chaos via duck solution breakdown in a piecewise linear van der Pol oscillator driven by an extremely small periodic perturbation," Physica D 194, 227 (2004).

${ }^{26} \mathrm{P}$. S. Landa and A. Rabinovich, "Exhibition of intrinsic properties of certain systems in response to external disturbances," Phys. Rev. E 61, 1829 (2000).

${ }^{27} \mathrm{X}$.-J. Wang, "Genesis of bursting oscillations in the Hindmarsh-Rose model and homoclinicity to a chaotic saddle," Physica D 62, 263 (1993).

${ }^{28}$ J. Moehlis, "Canards in a surface oxidation reaction," J. Nonlinear Sci. 12, 319 (2002).

${ }^{29}$ D. Setsinsky, A. Shishkin, L. Ryazanova, and D. Postnov, "Subthreshold oscillations alter noise-induced dynamics," Proc. SPIE 5696, 168 (2005). 